\title{
COMUNICACIÓN
}

\section{Presencia de Eustrongylides tubifex (Nematoda: Dioctophymatoidea) en la Patagonia, Argentina}

\author{
NORMA BRUGNI* y GUSTAVO VIOZZI*
}

\section{PRESENCE OF Eustrongylides tubifex IN ARGENTINIAN PATAGONIA}

Presence of an ovigerous female of Eustrongylides tubifex has been recorded from the proventriculus of Podiceps major from a Patagonian andean lake. Two new localities for Galaxias maculatus harbouring larvae of $\boldsymbol{E}$. tubifex are given. Prevalence of infection in lake Quillén is higher than previous reports for Lake Gutiérrez.

Key words: Eustrongylides tubifex, Podiceps major, Galaxias maculatus, Patagonia.

\section{INTRODUCCIÓN}

Tres especies del género Eustrongylides han sido registradas en aves piscívoras de distintas partes del mundo ${ }^{1}$ : E. ignotus, E. excisus y E. tubifex. El ciclo de vida de este nemátodo incluye oligoquetos acuáticos y peces como hospedadores intermediarios y aves ictiófagas como hospedadores definitivos. E. tubifex ha sido reportada en Gaviiformes, Anseriformes, Ciconiiformes y Podicipediformes en Estados Unidos, Canadá, Brasil, Europa, y Rusia ${ }^{2}$. Especies de este género pueden provocar mortalidades en aves ${ }^{3}$ y transmitirse al hombre mediante el consumo de pescado crudo o insuficientemente cocido ${ }^{4}$.

Estudios previos realizados en Galaxias maculatus (puyen chico) del lago Gutiérrez, Bariloche, Argentina ${ }^{5}$, mostraron la presencia de larvas del tercer y cuarto estadio de Eustrongylides sp. con baja prevalencia $(0,78 \%)$ e intensidad media ${ }^{1}$. Si bien las características morfológicas de las larvas recuperadas y del ejemplar subadulto obtenido de infecciones experimentales en pollos coincidían con las de E. tubifex ${ }^{6}$, la identidad específica no fue corroborada.
El objetivo del presente trabajo es informar la presencia E. tubifex en un ave autóctona de la Patagonia argentina y ampliar el listado de localidades para larvas en $G$. maculatus.

\section{MATERIAL Y MÉTODOS}

Entre septiembre de 1998 y agosto de 1999 se tomaron muestras mensuales en el lago Moreno $\left(41^{\circ} 04^{\prime} \mathrm{S} ; 71^{\circ} 33^{\prime} \mathrm{O}\right)$, capturándose 611 puyenes. Adicionalmente se tomó una muestra de 10 puyenes en el lago Quillén $\left(39^{\circ} 25^{\prime} \mathrm{S}\right.$; $71^{\circ} 20^{\prime} \mathrm{O}$ ) en enero de 2000 . Los peces fueron capturados con nasas cebadas, transportados al laboratorio y mantenidos vivos hasta su procesamiento. La observación macroscópica reveló la presencia de peces con cápsulas melanizadas en el pedúnculo caudal. De cada una de estas cápsulas se extrajeron larvas de Eustrongylides sp. En noviembre de 1999 se recogió un ejemplar muerto de Podiceps major en el lago Moreno, se disecó el tubo digestivo, observándose lesiones en los tejidos de la pared del proventrículo. Los parásitos extraídos de estas lesiones fueron fijados en formol $5 \%$ y diafanizados en lactofenol. Los huevos fueron

* Laboratorio de Parasitología, Centro Regional Universitario Bariloche, Universidad Nacional del Comahue, Quintral 1250 (8400) Bariloche, Argentina. Fax: +54-2944-422111. E-mail: nbrugni@crub.uncoma.edu.ar 
medidos en micrones (um) $(\mathrm{N}=25)$ registrándose el promedio y el rango entre paréntesis.

\section{RESULTADOS}

En el lago Moreno, de 51 peces capturados en el mes de marzo se encontró un puyen con cápsula melanósica en el pedúnculo caudal. En el muestreo estival del lago Quillén, de 10 puyenes capturados, tres tenían parásitos; uno dos larvas y el otro una larva, presentando todos los peces en ambas localidades una única cápsula melanósica en el pedúnculo caudal con larvas del tercer estadio.

Del examen de la pared del proventrículo de $P$. major, se recuperó una hembra con huevos. El extremo anterior presentó un círculo interno de seis papilas labiales con apéndices en forma de espina y un círculo externo de 6 papilas cefálicas más grandes con base amplia y ápices en forma de pezón (Figura 1). El extremo posterior presentó aspecto romo, con ano terminal y útero con huevos embrionados (Figura 2). Las medidas de los huevos fueron 91,9 $(84-103,2)$ de largo y 43,8 (40,8 - 48) de ancho (Figura 3).

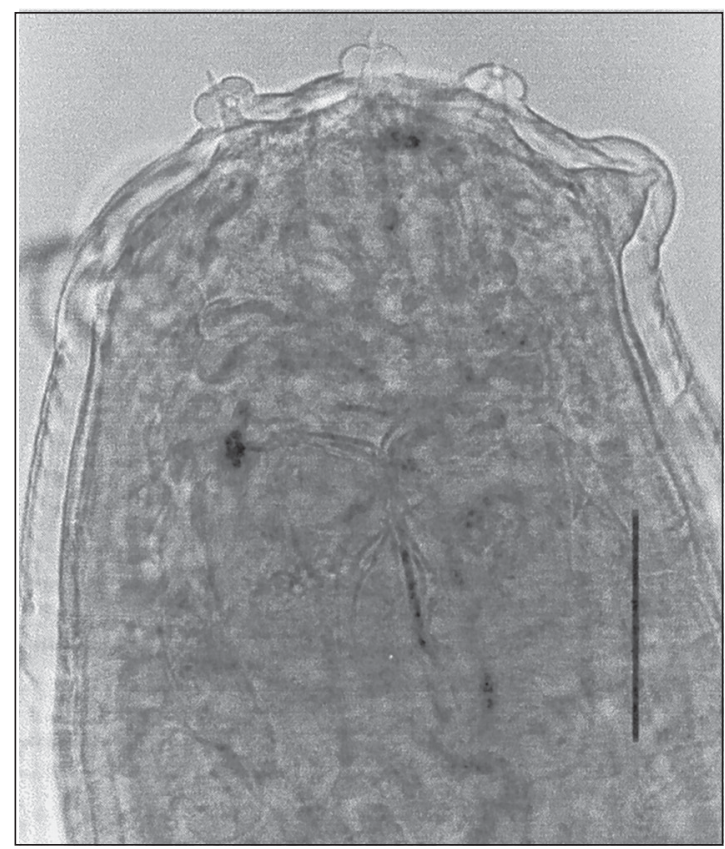

Figura 1. Extremo anterior de hembra adulta de Eustrongylides tubifex recuperada de Podiceps major. Barra $=100 \mu \mathrm{m}$.

\section{DISCUSIÓN}

Las características morfológicas del nematodo adulto recuperado de $P$. major en el lago Moreno corresponden con las descriptas para E. tubifex ${ }^{1}$.

Si bien las bajas prevalencias e intensidades de infección de este parásito en el lago Moreno concuerdan con los registros previos ${ }^{5}$, los valores y las características de la infección en el lago Quillén sugieren que la población de puyenes de este lago presentaría un patrón de infección con valores mayores de prevalencia e intensidad, siendo esta la primera vez que se registra la presencia de más de una larva de Eustrongylides sp. por cápsula en peces.

Un estudio de la fauna de oligoquetos acuáticos de la Patagonia reveló la presencia de hospedadores potenciales de E. tubifex como Limnodrilus hoffmeisteri y Tubifex tubifex ${ }^{7}$, los que, al igual que en el Hemisferio Norte ${ }^{8}$, participarían también como hospedadores intermediarios en lagos andino patagónicos.

El hallazgo de un ejemplar adulto en un hospedador natural permitiría inferir la identidad específica de las larvas de Eustrongylides sp.

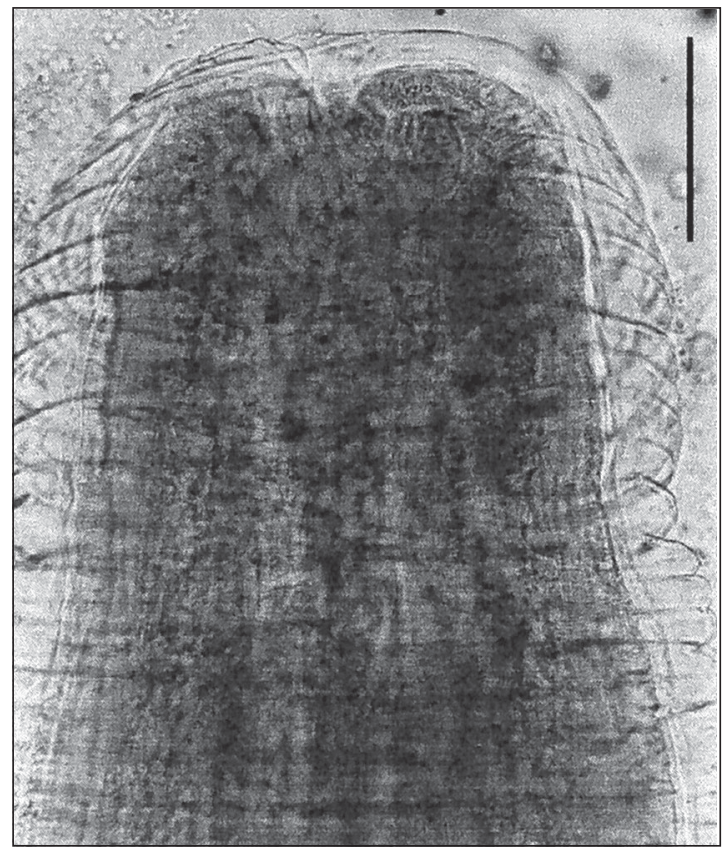

Figura 2. Extremo posterior de hembra adulta de Eustrongylides tubifex recuperada de Podiceps major. Barra $=200 \mu \mathrm{m}$. 


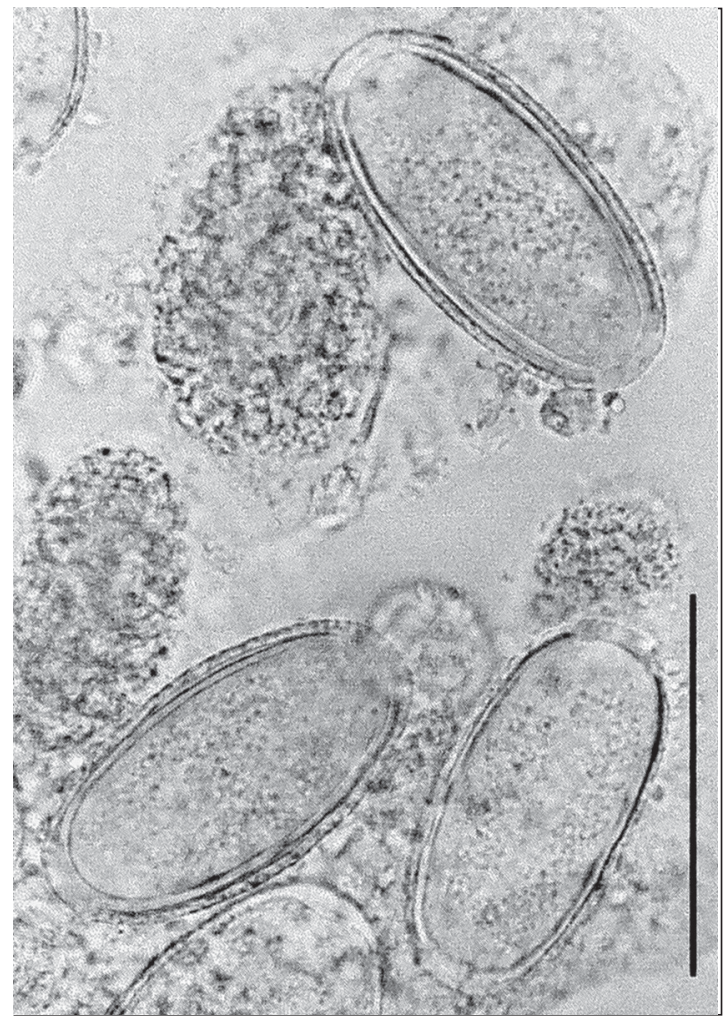

Figura 3. Huevos embrionados de hembra adulta de Eustrongylides tubifex recuperada de Podiceps major. Barra $=100 \mu \mathrm{m}$.

encontradas en el G. maculatus ${ }^{5}$, siendo éste el primer registro de una especie de este género para aves en la Argentina.

El registro de la presencia de una especie del género Eustrongylides reviste importancia desde el punto de vista zoosanitario, ya que factores como la contaminación de los cuerpos de agua pueden provocar la proliferación de las poblaciones de tubifícidos, propiciando las condiciones para el desarrollo de epizootias en aves ictiófagas ${ }^{9}$, así como el incremento del riesgo de transmisión al hombre a través del consumo de pescado ${ }^{4}$.

\section{RESUMEN}

La presencia de una hembra ovígera de Eustrongylides tubifex en el proventrículo de un Podiceps major se describe por prímera vez en un ave de un lago andino de la Patagonia Argentina. En dos nuevas localidades el parásito en Galaxias maculatus. La prevalencia de infección es mayor en el lago Quillén que la reportada previamente en el lago Gutiérrez.

\section{REFERENCIAS}

1.- MEASURES L N. Revision of the genus Eustrongylides Jägerskiöld, 1909 (Nematoda: Dioctophymatoidea) of piscivorous birds. Can J Zool 1988; 66: 885-95.

2.- FRIEND M, FRANSON C (Eds.). Field Manual of Wildlife Diesases: General Field Procedures and Diseases of Birds, Washington, 1999; 426 pp.

3.- SPALDING M G, FORRESTER D J. Pathogenesis of Eustrongylides ignotus. (Nematoda: Dictiophymatoidea) in Ciconiiformes. J Wild Dis 1993; 29: 25060.

4.- LICHTENFELS J R, STROUP C F. Eustrongylides sp. (Nematoda: Dioctophymatoidea): first report of an invertebrate host (Oligochaeta: Tubificidae) in North America. Proc. of the Helminthol Soc Wash 1985; 52: 320-3.

5.- BRUGNI N, VIOZZI G. Presence of Eustrongylides sp. (Jägerskiöld, 1909) (Nematoda: Dioctophymatoidea) in Galaxias maculatus (Jenyns, 1842) (Pisces: Galaxiidae) from Patagonia, Argentina. J Helminthol Soc Wash 1999; 63: 43-5.

6.- MEASURES L N. Epizootiology, pathology, and description of Eustrongylides tubifex (Nematoda: Dioctophymatoidea) in fish. Can J Zool 1988; 66: 2212-22.

7.- SEMENAS L. (Ed). Monitoreo de hospedadores potenciales de Myxobolus cerebralis en Patagonia, Argentina, Bariloche, 1999; 147 pp.

8.- MEASURES L N. The development of Eustron-gylides tubifex (Nematoda: Dioctophyma-toidea) in oligochaetes. J Parasitol 1988; 74: 294-304.

9.- SPALDING M G, BANCROFT G T, FORRESTER D J. The epizootiology of eustrongylidosis in wading birds (Ciconiiformes) in Florida. J Wild Dis 1993; 29: 237-49.

Agradecimientos: Fondos provistos por los subsidios CONICET. PICT-FONCYT 01-00002-00067 y UNC-B7020 